\title{
Animation of Magnetically Levitated Shoes and Its Optical Flow with Computer Vision
}

\author{
Kuldip Acharya ${ }^{\mathrm{a}}$, Dr. Dibyendu Ghoshal ${ }^{\mathrm{b}}$ \\ ${ }^{a}$ National Institute of Technology Agartala, Computer Science and Engineering Dept., Barjala, Jirania, West \\ Tripura, Pin: 799055, INDIA \\ ${ }^{b}$ National Institute of Technology Agartala, Department of Electronics and Communication Engineering, \\ Barjala, Jirania, West Tripura, Pin: 799055, INDIA
}

Received: 06 July 2017; Accepted: 08 January 2018; Published: 08 May 2018

\begin{abstract}
The article presents a concept of computer-aided design and three-dimensional (3D) computer animation of a newly proposed magnetically levitation based shoes where the users can move in the air. The aerial movement would be in the direction of a magnetic track which is laid down below the trajectory and the users have to wear the maglev shoes. They can move from ground floor to upper floors as in the case of an elevator. The users have been provided adequate control over the speed of movement and they can stop and run the system by themselves at any instant of time by self-controlling of the motion generated by them. The maglev shoes are proposed to be built with superconducting materials to levitate above the magnetic tracks. Computer vision features are efficiently utilized to detect various features of the animated image frame of maglev shoes and the motion study of the proposed system. Statistical methods with existing functions are used to analyze various features like speed, angles and finding the optical flow in both horizontal and vertical direction of maglev shoe wearers.
\end{abstract}

Index Terms: Autodesk Maya, 3D animation, computer vision, magnetic levitation.

(C) 2018 Published by MECS Publisher. Selection and/or peer review under responsibility of the Research Association of Modern Education and Computer Science.

\section{Main Text}

Three-dimensional (3D) modeling and computer animation of magnetically levitated shoes are done in the animation software, Autodesk Maya 2016 student version [1]. Computer graphics and animation can offer some insight into any physical entity before the actual physical implementation of it. The magnetic levitation * Corresponding author. Tel.: 9774554823

kuldip.acharjee@gmail.com 
shoes are proposed to be designed with type II superconductors [2]. The type II superconductors have the ability to operate at a much higher critical temperature than type I superconductors [3]. The proposed maglev shoe operates on the basis of the electrodynamic type of superconductivity. The strength of the levitation force depends on the magnitude of the current flowing through the corresponding superconductor related circuits and systems. The phase of the current play vital roles in controlling the accuracy of the levitation path followed by the users of the shoe. The proposed shoes are expected to offer the users a new adventure while moving from floor to floor. This can be displayed in the 3D model and animation done in the animation software Autodesk Maya 2016 student version and has been incorporated in the present paper. Besides this computer vision system is utilized to study the whole maglev system. We have utilized various optical flow algorithms to monitor the system and to track the user movements. It gives a clear picture in which direction the user is actually moving. We have compared various optical flow algorithms. The simulation results return the running time of each algorithm and thus shows which algorithm is the best. Thus, computer vision algorithm utilized to monitor the speed and direction of user movement, track and to improve the performance of overall maglev shoes.

\section{Related Works}

\subsection{Review of Superconductors, its Application, and its Advantages and Disadvantages}

A superconductor [4] is a special type of material that ideally conducts electricity without resistance. That means that electrons can flow very easily through the material. This can be achieved only at very low temperatures. A superconductor can carry a current indefinitely without losing any energy. This property creates the possibility of frictionless, high-speed transportation models such as maglev trains [5], as well as friction-free bearings and flywheels. A superconductor can cause a magnet to levitate or float above it because it repels the magnetic field. Superconducting Wires [6] can be made from niobium-tin (NB3SN) have a BC2 as high as 24.5 Tesla. This makes them useful for applications requiring high magnetic fields, such as magnetic resonance imaging (MRI) machines. The advantage of using superconducting electromagnets is that the current only has to be applied once to the wires, which are then formed into a closed loop and allow the current to persist indefinitely - as long as the superconductor stays below the critical temperature. Even if, the external power supply is switched off, current can still flow through it. Superconductors offer numerous advantages compared with ordinary conductors. The first advantage is power and cost efficiency, which is due to the negligible energy losses that occur in superconductors. The effectiveness of superconductors also leads to environmental benefits, including reduced fuel consumption and carbon dioxide emissions that result from lower electricity losses. Superconductor technology will contribute to reducing the size and weight of motors, generators, and supporting equipment. The only disadvantage of a superconductor is the cost of the superconducting coils cooling system.

\subsection{Optical Flow Farneback class[7]}

The Farneback technique is employed to work out the direction and speed of a moving object from one image or video frame to different images or video frames.

\section{Description}

Optical Flow $=$ Optical Flow Farneback provides the direction associated speed of the corresponding object in motion which is calculated by an optical flow object. The estimate flow technique of the Farneback formula is used to estimates the optical flow.

\section{3 optical Flow HS class [8]}


The Horn-Schunck method is utilized to calculate the speed and direction of an object in motion from one image or video frame to another image or video frame.

$I_{x} u+I_{y} v+I_{t}=0$

$I_{x}, I_{y}$, and $I_{t}$ are the spatiotemporal image brightness derivatives.

$u$ denoted the horizontal optical flow.

$v$ denoted the vertical optical flow.

\subsection{Horn-Schunck Method [8]}

$$
E=\iint\left(I_{x} u+I_{y} v+I_{t}\right)^{2} d x d y+\alpha \iint\left\{(\partial u / \partial x)^{2}+(\partial u / \partial y)^{2}+(\partial v / \partial x)^{2}+(\partial v / \partial y)^{2}\right\} d x d y
$$

Here, $\partial u / \partial x$ and $\partial u / \partial y$ are the spatial derivatives of the optical velocity component, $u$. The global smoothness term is tuned by $\alpha$. The velocity field, $[u v]$, for each pixel in the image is obtained by using the Horn-Schunck method algorithm. [1]

\subsection{Lucas-Kanade Method [8]}

The Lucas-Kanade methodology divides the first image or video frame into smaller sections to see optical flow for $\mathrm{u}$ and $\mathrm{v}$. It assumes a relentless speed in every section of an image.

\subsection{Optical Flow LK DoG class [8]}

The direction and speed of a moving object from a video frame or one image to another image are calculated utilizing the Lucas-Kanade DoG method. The Lucas-Kanade method and a derivative of Gaussian (DoG) filter are utilized for temporal smoothing.

\section{Proposed Design of Magnetically Levitated Shoe}

Figure 1, shows various components of the magnetically levitated shoe. The proposed shoe, as illustrated in figure 1, may be developed by using the materials like cotton, leathers, rubber and plastic materials. Rubber plays a major role in the construction of these shoes. When a wearer puts his legs in the shoes, then due to the flexibility of soft rubber, the shoes can easily grow or shrink and fit into their feet. In the magnetically levitated shoes, superconducting materials are used on the bottom side of the shoes and to protect the passenger from the chill of cryogenic temperature [9] a shielding material, such as lightweight insulator layer can be used between the feet resting layer and superconducting layer. The additional purpose of using such shielding layer is to protect a wearer having either a cardiac pacemaker or other similar gadgets which are prone to be affected by cryogenic environment and superconductivity. The superconductor layer at the bottom part of the shoe can be created by injecting liquid nitrogen through a nozzle fitted with a sealed lead provided with a screwing arrangement and in the tubular container, liquid nitrogen is kept. Type II superconductor is used here to levitate the shoes above the magnetic track. In a cryogenic temperature, a conductor loses its resistance. In type II superconductor an object levitates not due to Meissner effects [10]. A disc of 3 inches diameter may carry approximately 70,000 times more weight than its own weight [11]. 
Proposed Shoe designed by materials like rubber, leathers, cottons and plastics.

Superconductor shielding thermocol

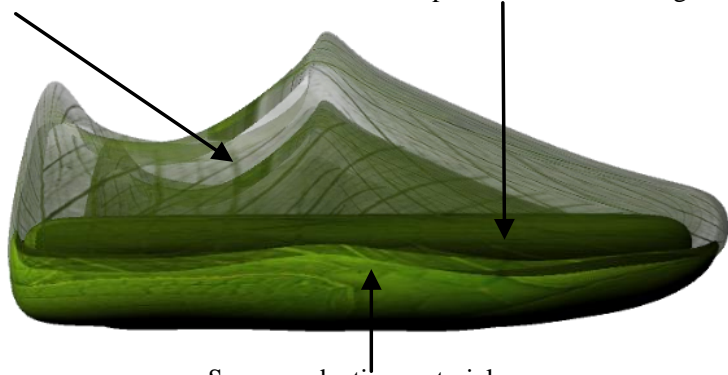

Superconducting material.

Fig.1. Different Components of Magnetically Levitate Shoes

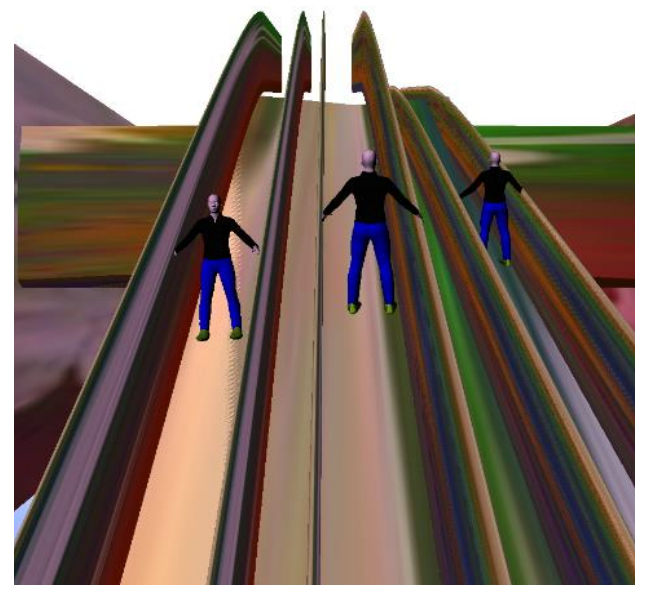

Fig.2. Passenger Travelling Scenario by Maglev Shoe

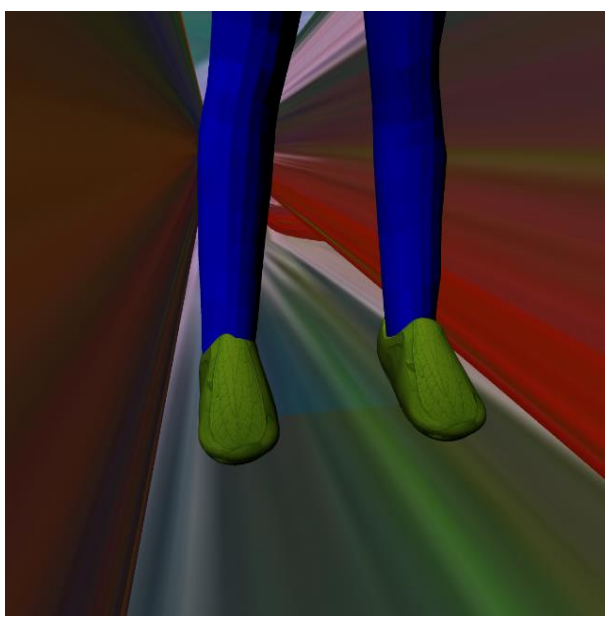

Fig.3. Close view of a Wearer Wearing the Maglev Shoes 
Passenger traveling scenario by Maglev shoe is shown in figure 2. The figure shows the movement of a passenger wearing the magnetically levitated shoe. Insulator material, superconductor material, and normal hoe layer would be designed such that all layers should have compatible dimension and fits in the proposed structure of the magnetically levitated shoe. The figure 3, shows the close view of magnetic levitating shoes. The wearers move from one place to the other place by walking in the air. They push themselves to start to levitate in the air. Little force is required to move from upward to downward in case of lower floor to upper floor movement when the wearer moves along any elevator made up of as follows. The elevator track may be made up especially with the set of strong permanent magnets or by the set of electromagnets. Besides magnetic part, other materials like steel, woods and rubber grips may be used to build the physical system for floating by the proposed magnetically levitating shoes. The wearer always needs to put their hands on the side walls gripping handle to avoid an accident. They can stop themselves. Figure 4, shows the different components of the magnetically levitated shoes.

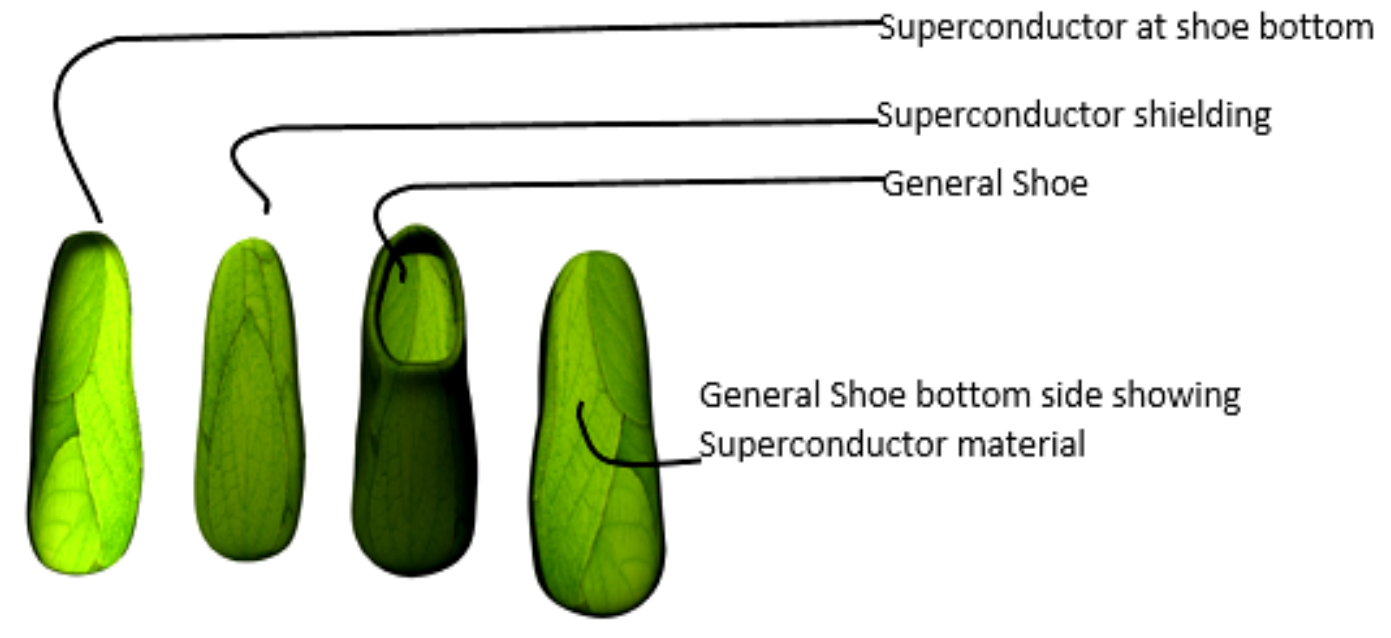

Fig.4. Different Parts of the Maglev Shoe

The superconducting layer is fitted at the bottom-most layer of the shoe. The superconducting shielding layer is placed above the superconductor layer. A movable handle is provided as a safety measure during the passage by the users of maglev shoes. The handle function same as an ordinary mechanical escalator. This would provide better safety to the users.

\section{Superconducting Cooling System}

In the present system, the cryogenic chip is used in the maglev shoes. The function of the maglev shoe depends on the position of a solid high-temperature superconductor. This type of superconductor is available which are found to be chip and it is minuscule in size. The chip can be used in the side wall of the shoe which is made of plastic, rubber, foam etc. The chip is operated by a small 5-volt battery as used in the pocket calculator. Any part of the system can be detached easily and can be repaired and it may be considered as an advantage. In the proposed system, the maintenance cost is expected to be very low although the infrastructure cost is very high. 


\subsection{Tables}

Table 1. Magnetically Levitating Shoe Components

\begin{tabular}{lll}
\hline Components & Proposed material used & Cost \\
\hline General Shoe & Leather, rubber, cotton1 & low \\
Superconductor shielding & Superconducting shield & low \\
Superconductor & Yttrium-barium-copper-oxygen ceramic & high \\
Superconducting shield & Wood, Thermo cool & low \\
\hline
\end{tabular}

Table 2. Materials used to Build the Magnetic Track.

\begin{tabular}{lll}
\hline Components & Magnetic field & Material used \\
\hline $\begin{array}{l}\text { Components are used to build the walker track is Iron, cobalt, and nickel. } \\
\text { Because it may use a Strong permanent magnet. }\end{array}$ & $\begin{array}{l}\text { Approx. 1.4 } \\
\text { teslas. }\end{array}$ & $\begin{array}{l}\text { Alloys of rare earth } \\
\text { elements. }\end{array}$ \\
\hline
\end{tabular}

Table 3. Proposed Materials used to build the Magnetic Track. [12]

\begin{tabular}{lll}
\hline Components & Description / full form & Discovered \\
\hline YBCO & Yttrium barium copper oxide. & In April 1986, Georg Bednorz and Karl Müller. \\
BSCCO & Bismuth strontium calcium copper oxide. & Around 1988 by Hiroshi Maeda and colleagues. \\
\hline
\end{tabular}

Table 4. Components used to make High-Temperature Superconducting Wire

\begin{tabular}{lll}
\hline Components & Description / full form & Discovered \\
\hline Walker height width and breath & Yttrium barium copper oxide. & In April 1986, Georg Bednorz and Karl Müller. \\
Walker angle of inclination & 26-30 degree. & Around 1988 by Hiroshi Maeda and colleagues. \\
\hline
\end{tabular}

Table 5. Components may use to make the Magnetic Levitate Shoe [13]

\begin{tabular}{lll}
\hline Shoe components & Materials used & Function \\
\hline Vamp & leather & $\begin{array}{l}\text { The segment of upper that covers the front of the foot similarly as the back as the } \\
\text { join of the quarter. } \\
\text { Makes a layer between the sole and the wearer's foot and it gives solace to the } \\
\text { wearer. }\end{array}$ \\
Rubber & $\begin{array}{l}\text { The uncovered piece of the sole that is contacted with the ground. Likewise, with } \\
\text { all parts of the shoe, outsoles are produced using a mixture of materials. The } \\
\text { properties of the outsole need are grasp, toughness, and water resistance. }\end{array}$ \\
Qutsole & $\begin{array}{l}\text { The back and sides of the upper that covers the heel that is behind the vamp. The } \\
\text { heel segment of the quarter is regularly reinforced with a stiffener, which helps } \\
\text { bolster the back of the foot. Some shoe outlines utilize a constant bit of calfskin for } \\
\text { the vamp and quarter. } \\
\text { The Mudguard is the shoe example part of the forward piece of the shoe along the } \\
\text { edge of the outsole. }\end{array}$ \\
Mudguard & Leather & $\begin{array}{l}\text { An infusion shaped a bit of plastic that gives the heel of the running shoe a firm } \\
\text { supporting feel. }\end{array}$ \\
\hline Reel stabilizer & Rubber and &
\end{tabular}


Table 6. SURF Features with 10 Strongest Points in $\mathrm{x}$ and Y-Direction

\begin{tabular}{cc}
\hline $\mathrm{x}$ coordinate & $\mathrm{y}$ coordinate \\
\hline 1.0100 & 0.2021 \\
1.0607 & 0.1096 \\
1.0967 & 1.0005 \\
1.0685 & 1.0640 \\
1.1779 & 0.1976 \\
1.0349 & 1.0630 \\
0.9832 & 0.2133 \\
1.1341 & 0.0380 \\
1.0829 & 0.3347 \\
0.8602 & 0.2108 \\
\hline
\end{tabular}

Table 7. The Actual Path and Tolerable Bending Angles

\begin{tabular}{cc}
\hline Actual angles & Tolerable bending angles \\
\hline $90^{\circ}$ & $93^{0}-87^{0}$ \\
$95^{\circ}$ & $92^{0}-98^{0}$ \\
$100^{\circ}$ & $87^{0}-97^{0}$ \\
$80^{\circ}$ & $83^{0}-77^{0}$ \\
\hline
\end{tabular}

Table 8. Maximum Bearable Weight

\begin{tabular}{cccc}
\hline Passenger weight & Freight weight & Total weight & Average weight \\
\hline 60 K.G. & 10 K.G. & 70 K.G. & \\
70 K.G. & 20 K.G. & 90 K.G. & 71.25 K.G \\
75 K.G. & 5 K.G. & 90 K.G. & \\
30 K.G. & 15 K.G. & 35 K.G. & \\
\hline
\end{tabular}

Table 9. Runtime of Different Optical Flow Algorithms

\begin{tabular}{ccccc}
\hline $\begin{array}{c}\text { Video } \\
\text { File }\end{array}$ & $\begin{array}{c}\text { Time Elapsed } \\
\text { for Farneback }\end{array}$ & $\begin{array}{c}\text { Time Elapsed } \\
\text { for Horn- } \\
\text { Schunck }\end{array}$ & $\begin{array}{c}\text { Time Elapsed for } \\
\text { Lucas-Kanade }\end{array}$ & $\begin{array}{c}\text { Time Elapsed for } \\
\text { Lucas-Kanade DoG }\end{array}$ \\
\hline Shoe1.avi & $44.6366 \mathrm{sec}$. & $20.3708 \mathrm{sec}$. & $6.1392 \mathrm{sec}$. & $29.2789 \mathrm{sec}$. \\
Shoe2.avi & $60.5439 \mathrm{sec}$. & $13.6559 \mathrm{sec}$. & $6.917 \mathrm{sec}$. & $33.9659 \mathrm{sec}$. \\
Shoe3.avi & $93.5649 \mathrm{sec}$. & $24.3135 \mathrm{sec}$. & $12.7166 \mathrm{sec}$. & $60.253 \mathrm{sec}$. \\
Shoe4.avi & $114.1614 \mathrm{sec}$. & $28.2581 \mathrm{sec}$. & $14.4434 \mathrm{sec}$. & $69.9993 \mathrm{sec}$. \\
Shoe5.avi & $59.0916 \mathrm{sec}$. & $16.505 \mathrm{sec}$. & $6.1392 \mathrm{sec}$. & $41.3221 \mathrm{sec}$ \\
\hline
\end{tabular}

Table 10. Horn-Schunck Algorithm Smoothing Values

\begin{tabular}{ccc}
\hline Algorithm & Smoothness & Runtime \\
\hline Horn-Schunck & 0.3 & $1.5041 \mathrm{sec}$. \\
& 0.5 & $2.8477 \mathrm{sec}$. \\
& 0.7 & $1.4082 \mathrm{sec}$. \\
& 0.9 & $1.4 \mathrm{sec}$. \\
\hline
\end{tabular}


Table 11. Farneback Algorithm Different Pyramid Levels

\begin{tabular}{ccc}
\hline Algorithm & Number of Pyramid Levels & Runtime \\
\hline \multirow{3}{*}{ Farneback } & 3 & $4.4861 \mathrm{sec}$. \\
& 5 & $9.2122 \mathrm{sec}$. \\
& 7 & $4.4993 \mathrm{sec}$. \\
\hline
\end{tabular}

Table 12. Lucas-Kanade Algorithm Different Noise Threshold

\begin{tabular}{ccc}
\hline Algorithm & Noise Threshold & Runtime \\
\hline & 0.03 & $0.71088 \mathrm{sec}$. \\
Lucas-Kanade & 0.05 & $1.1642 \mathrm{sec}$. \\
& 0.07 & $0.67474 \mathrm{sec}$. \\
& 0.09 & $0.68228 \mathrm{sec}$. \\
\hline
\end{tabular}

Table 13. Lucas-Kanade Dog Algorithm Different Noise Threshold

\begin{tabular}{ccc}
\hline Algorithm & Noise Threshold & Runtime \\
\hline & 0.03 & $2.8251 \mathrm{sec}$. \\
Lucas-Kanade DoG & 0.05 & $5.0657 \mathrm{sec}$. \\
& 0.07 & $2.6994 \mathrm{sec}$. \\
& 0.09 & $2.7181 \mathrm{sec}$. \\
\hline
\end{tabular}

\section{A Study on Maglev Shoes with Computer Vision}

The speeded up robust features (SURF) [13] function is utilized in animated video frames to discover useful information and extracting the features of proposed Maglev shoes. The areas of interest have been shown at that point selecting the strongest features of the video frames and plot it. We can see here five most grounded elements have been appeared in figure 5(a)(b), to compute optical flow to estimate the direction and speed of Maglev shoe wearer moving from one location to another is done using the Horn-Schunck method. Optical flow estimates the direction and speed of moving object both from one picture to another or from one video frame to another video frames. The video of the animation is designed in the Autodesk Maya software. The total time elapsed is 113.8846 seconds. The maximum angle is obtained to be 176.1098 degrees and up to 360 angles are added to get the edges in positive values. In this way, 360-176.11=183.89 degrees are obtained. The minimum angle is 180 degrees. The calculated magnitude is obtained from the following equation:

$$
\text { Magnitude }=\operatorname{sqrt}(\mathrm{Vx} . * \mathrm{Vx}+\mathrm{Vy} . * \mathrm{Vy})
$$

'Vx' - X component of velocity. 'Vy' — Y component of velocity. 'Magnitude' — Magnitude of optical flow [14].

Few major parameters determine the accuracy of the levitation trajectory. The phase change in the current can lead to a change in the angular position of the shoe wearer. The weight of the shoe wearer will be given by the following equation:

P. E=mgh

Where $\mathrm{m}=$ mass of the wearer $\mathrm{g}=$ acceleration due to gravity

$\mathrm{h}=$ height of the shoe wearer from the ground plane. 


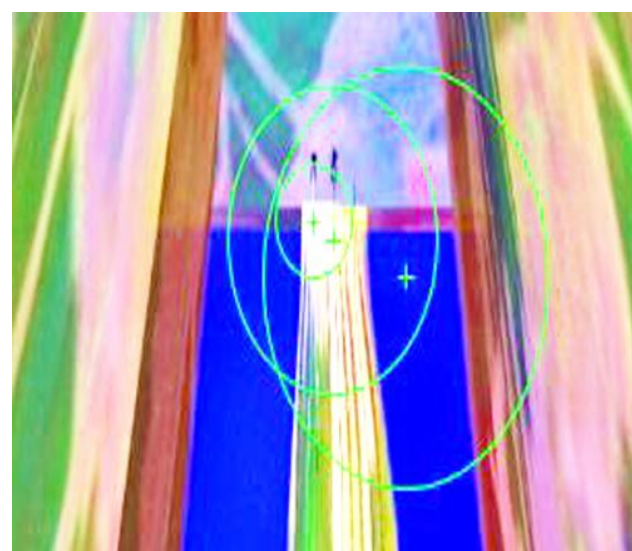

Fig.5(a). Surf Features of Maglev Shoes Animation

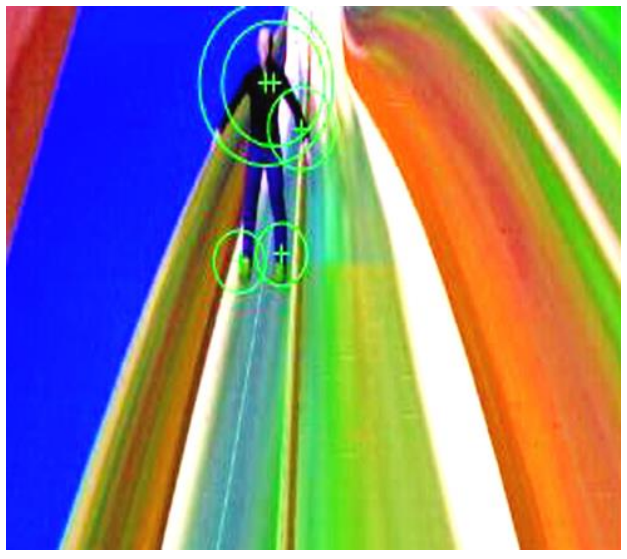

Fig.5(b). Surf Features of Maglev Shoes Animation

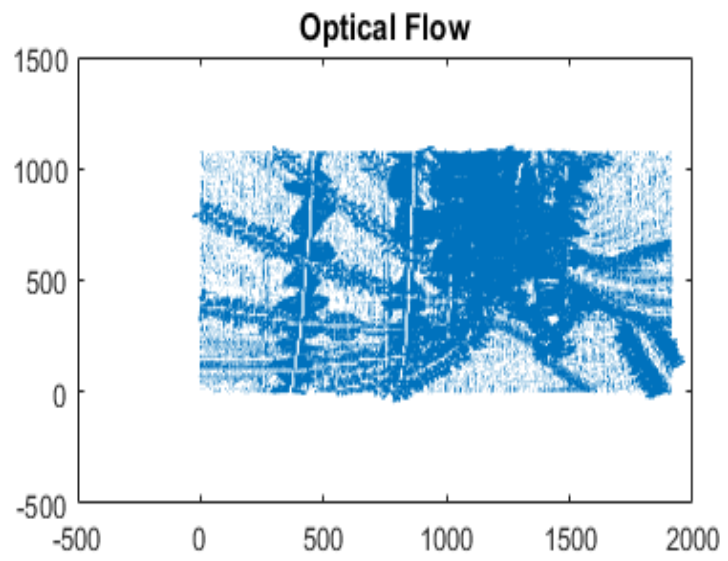

Fig.6. Example of Optical Flow

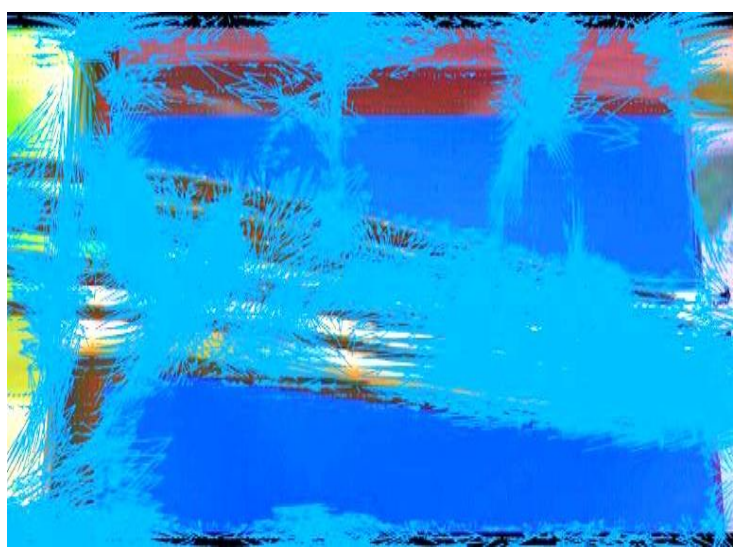

Fig.7. Another Example of Optical Flow 
The wearer is supposed to change his/her path (i.e. trajectory) by his/her own. The deviation of the linear path, angular direction, bending etc. will have to be limited to a tolerable limit. It is sufficient to assume from intuition the deviation should be limited to $\pm 5 \%$ of the assigned value. The normal practice to the angle should be $90^{\circ}$. The bending angle may be $85.5^{\circ}$ to $94.5^{\circ}$. Figure $11-14$ shows different runtime obtained based on the different smoothing values of the given parameters in the tables 10-13. We have used different tuneable values for smoothness in the Horn-Schunck algorithm, different Number of Pyramid Levels in the Farneback algorithm, different noise threshold in the Lucas-Kanade algorithm and Lucas-Kanade DoG algorithm. It is observed that Lucas-Kanade algorithm gives the best result.

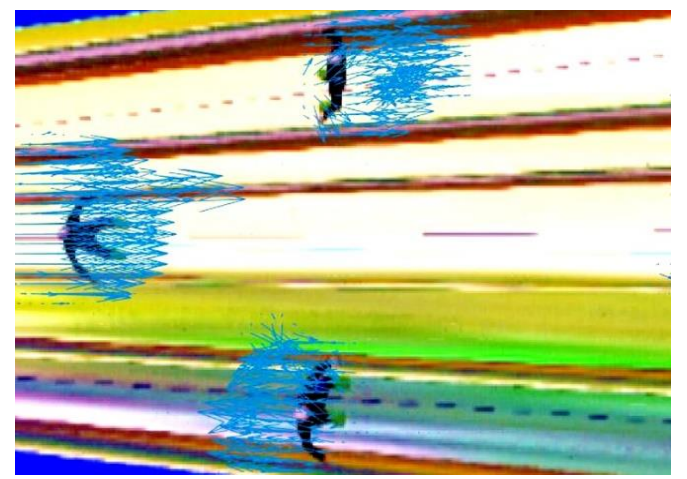

Fig.8. Optical Flow Moving in the Right Direction

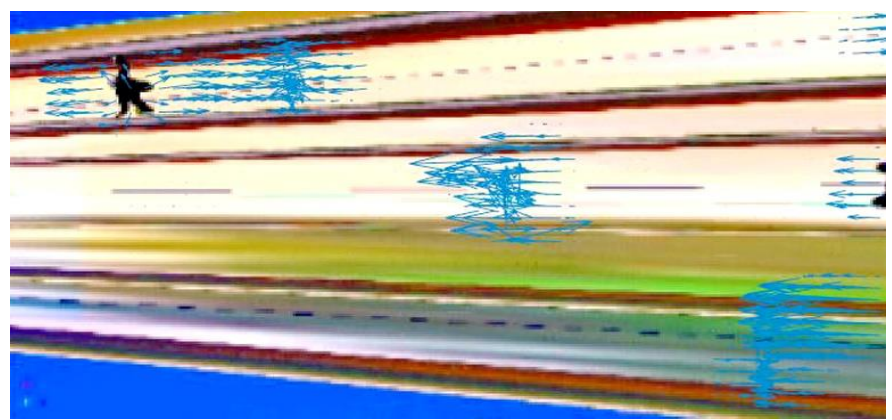

Fig.9. Optical Flow Moving in the Left Direction

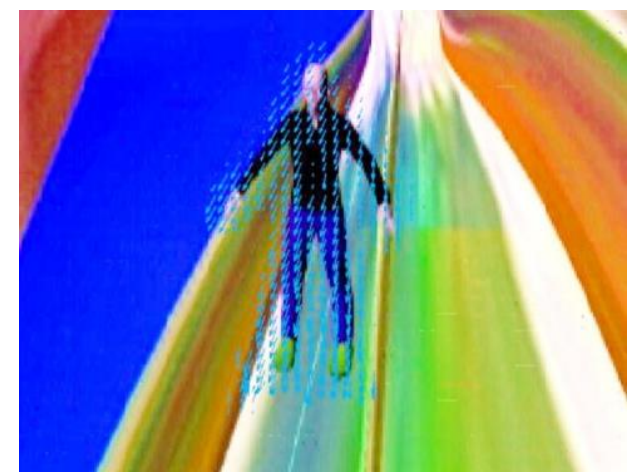

Fig.10. Optical Flow Example 


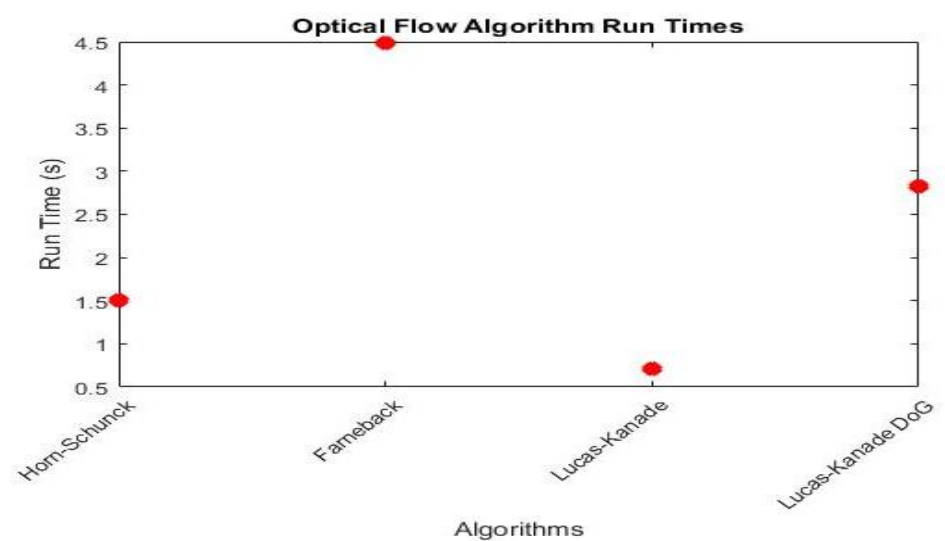

Fig.11. Run Time when Smoothness Value Is 0.3

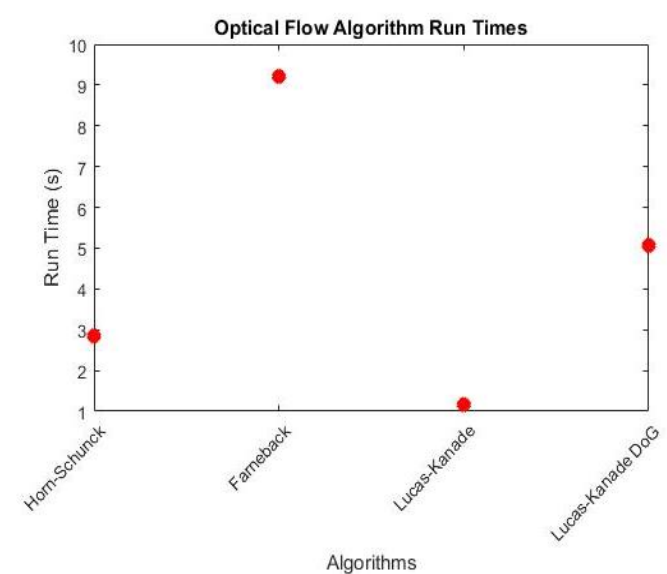

Fig.12. Run Time when Smoothness Value is 0.5

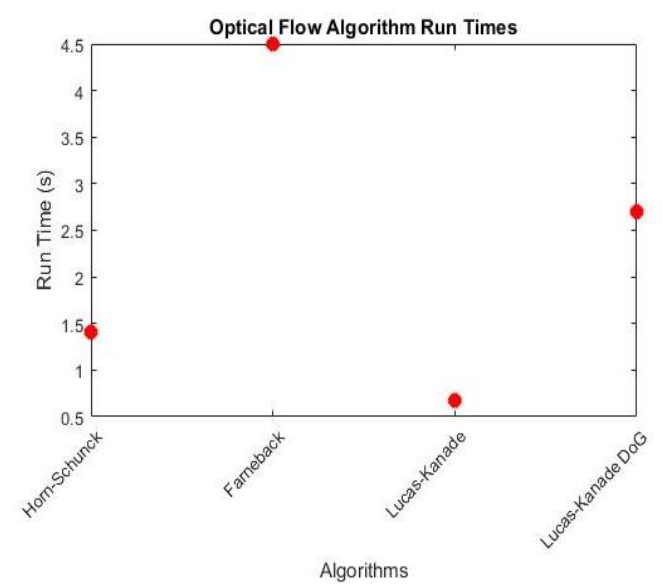

Fig.13. Run Time when Smoothness Value Is 0.7 


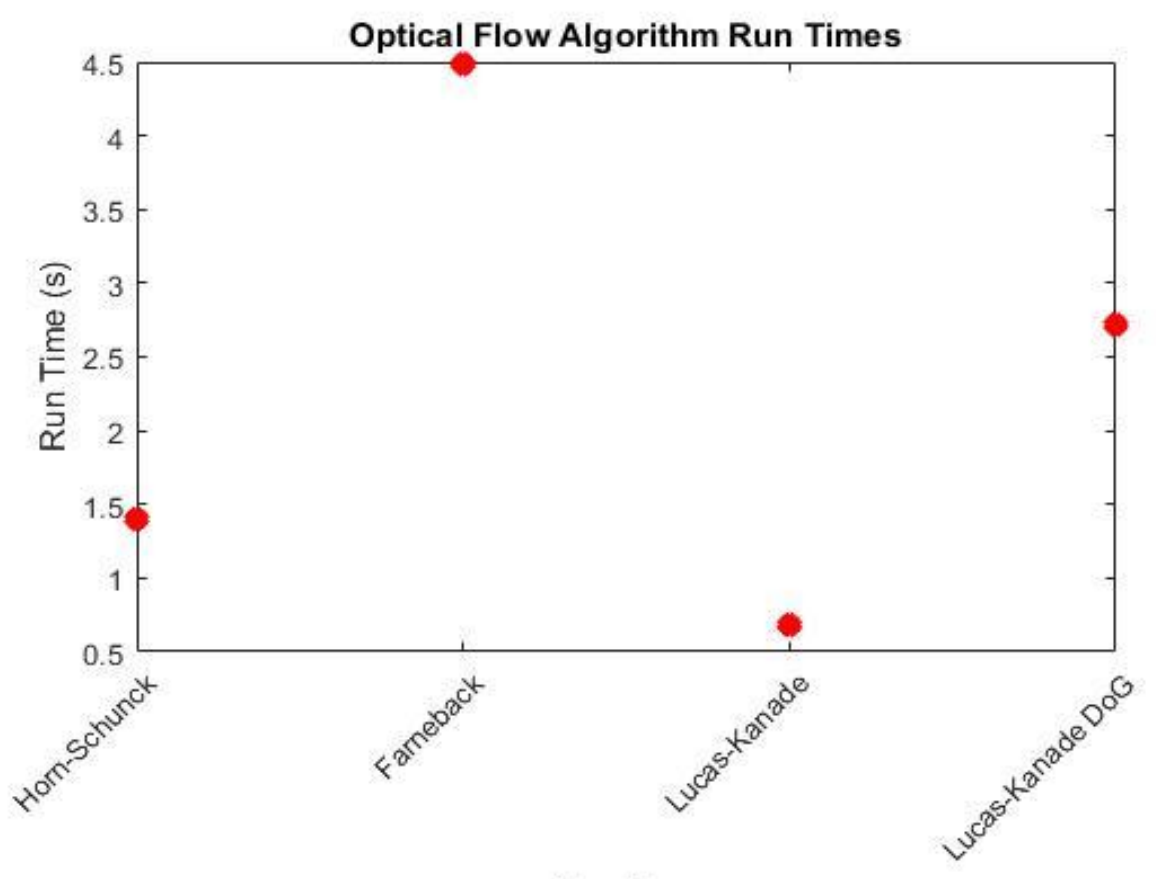

Algorithms

Fig.14. Run Time when Smoothness Value is 0.9

\section{Conclusion}

The proposed study on maglev shoe may be expected to find some utility in the real world. The idea can be more easily implementable for persons with smaller body weight. The choice of a solid high-temperature superconductor is very critical for the design of the shoe. The study of computer vision on magnetically levitated shoes using optical flow gives results like its velocity, trajectories, angles of motion, SURF features with the strong feature point of the maglev shoe wearers. Statistical methods are utilized with existing features and various results are found about the animation enhancement and motion-related information of the proposed system. The animated version of the shoe movement has been found to be capable of exhibiting the real-life use of shoes aiding the users to move in the air.

\section{Acknowledgment}

The first author acknowledges his gratitude to his supervisor Dr. D. Ghoshal and National Institute of Technology Agartala, India for helping and inspiring to do the Ph.D. research work.

\section{Dedication}

Author one dedicates his research work to his loving father Dr. Kalidas Acharya. 


\section{References}

[1] Derakhshani, Dariush. Introducing Autodesk Maya 2013. John Wiley \& Sons, 2012.

[2] Goodman, b. b. "type II superconductors." reports on progress in physics 29.2 (1966): 445.

[3] Type I superconductors http://hyperphysics.phy-astr.gsu.edu/hbase/solids/scond.html

[4] Superconductor, http://hyperphysics.phy-astr.gsu.edu/hbase/solids/scond.html

[5] Hyung-woo, ki-chan kim, and ju lee. "review of maglev train technologies." ieee transactions on magnetics 42.7 (2006): 1917-1925.

[6] Kang, Sukill, et al. "High-performance high-Tc superconducting wires." Science 311.5769 (2006): 1911-1914.

[7] Farneback, G. "Two-Frame Motion Estimation Based on Polynomial Expansion." Proceedings of the 13th Scandinavian Conference on Image Analysis. Gothenburg, Sweden, 2003.

[8] Barron, J. L., D. J. Fleet, S. S. Beauchemin, and T. A. Burkitt. "Performance of optical flow techniques". CVPR, 1992.

[9] Bhadrachalam, Pradeep, et al. "Energy-filtered cold electron transport at room temperature." Nature Communications. 5, 4745 (2014).

[10] Alff, L., et al. "Observation of bound surface states in grain-boundary junctions of high-temperature superconductors." Physical Review B 58.17 (1998): 11197.

[11] Anatomy of the shoe by admin. http://www.shoeguide.org/shoe_anatomy. Date accessed: July 06/2017.

[12] Alff, L., et al. "Observation of bound surface states in grain-boundary junctions of high-temperature superconductors." Physical Review B 58.17 (1998): 11197.

[13] Bay, H., A. Ess, T. Tuytelaars, and L. Van Gool. "SURF: Speeded Up Robust Features." Computer Vision and Image Understanding (CVIU). Vol. 110, No. 3, pp. 346-359, 2008.

[14] Optical Flow class, https://in.mathworks.com/help/vision/ref/opticalflow-class.html. Date accessed: July 06/2017.

\section{Authors' Profiles}

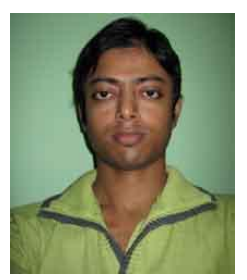

Kuldip Acharya received his M.Tech in CSE from Tripura University, Tripura, India in 2012. He is doing Ph.D. in Computer Science \& Engineering from National Institute of Technology Agartala, India from 2013. His areas of interest are computer vision, 3D Computer Animation \& design and, image processing.

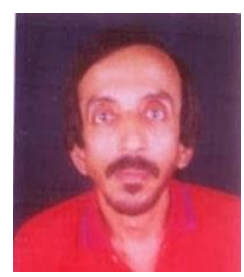

Dibyendu Ghoshal has received his B.Sc. (Honors in Physics), B.Tech \& M.Tech in Radiophysics and Electronics, all from Calcutta University(CU) in 1981, 1985 and 1987 respectively. He joined Indian Engineering Services (Group-A) in 1988 and served Department of Telecommunication, GOI. After holding various posts for three years, D. Ghoshal left the job to pursue higher studies and was awarded SRF in 1992 by CSIR. Subsequently, he was awarded Postdoctoral Research Associateship in 1996 and Ph.D. in Radiophysics \& Electronics from CU in 1997 with specialization in Microwave and millimeter wave systems. D. Ghoshal has served as a post-doctoral research associate and scientist for nearly 12 years and was associated with various projects sponsored by GOI. His research interest includes micro \& 
millimeter wave, semiconductor physics \& devices, Digital Image Processing, computer vision and computer animation.

How to cite this paper: Kuldip Acharya, Dibyendu Ghoshal,"Animation of Magnetically Levitated Shoes and Its Optical Flow with Computer Vision", International Journal of Engineering and Manufacturing(IJEM), Vol.8, No.3, pp.40-53, 2018.DOI: 10.5815/ijem.2018.03.04 